\title{
Aves en un humedal marino del sur de Chile
}

\author{
Birds in a marine wetland of southern Chile \\ Jaime A. Cursach ${ }^{1}$, Jaime R. Rau ${ }^{1}$ y Claudio N. Tobar ${ }^{1}$ \\ ${ }^{1}$ Laboratorio de Ecología \& Programa IBAM, Universidad de Los Lagos, Casilla 933, Osorno, Chile. jcurval@gmail.com
}

\begin{abstract}
This study evaluates the influence of tidal cycle, time-of-day and season on the diversity of a bird assemblage in the marine wetland of Lenca Bay in Reloncaví Sound, southern Chile. The study was carried out during four seasons, spring 2006, summer, fall and winter 2007. During all the study period, it was recorded 2502 birds belonging to 35 species and 19 families. The family Laridae had the highest species richness. Three species were the most relevant according to a relative abundance index for the assemblage of studied birds, Larus dominicanus, Larus maculipennis and Cygnus melancorypha. The highest number of bird species and abundance was recorded during summer. The diversity values were high in low tide, due to the entry of birds belonging to the "agroecosystems" and systems of shrub vegetation adjacent to marine wetland, terrestrial species as Milvago chimango, Vanellus chilensis, Theristicus melanopis, Polyborus plancus and passerine birds. The diversity values obtained between the three daily period of observation shows no significant differences. Finally we identified some human activities that threaten the conservation of birds in Reloncaví Sound.
\end{abstract}

Key words: Tidal cycle, time-of-day, seasonal variability, conservation

Resumen. - Este estudio evalúa la influencia del ciclo mareal, la hora del día y la estación climática sobre la diversidad del ensamble de aves presentes en el humedal marino de Bahía Lenca en el Seno de Reloncaví, sur de Chile. Las observaciones del estudio fueron realizadas durante las estaciones de un ciclo anual, primavera 2006, verano, otoño e invierno 2007. Durante todo el periodo de estudio, se registraron 2502 aves pertenecientes a 35 especies y 19 familias. La familia Laridae presentó la mayor riqueza de especies. Tres especies fueron las más relevantes de acuerdo a un índice de importancia relativa para el ensamble de aves observado: Larus dominicanus, Larus maculipennis y Cygnus melancorypha. Durante el verano se registró el mayor número de especies y abundancia de aves. Los valores de diversidad fueron significativamente mayores en los momentos de marea baja, debido al ingreso de aves pertenecientes a los "agroecosistemas" y sistemas de vegetación arbustiva colindantes al humedal marino, como las especies terrestres Milvago chimango, Vanellus chilensis, Theristicus melanopis, Polyborus plancus y aves Passeriformes. Los valores de diversidad obtenidos entre los tres horarios de observación no presentaron diferencias significativas, durante todo el período de estudio. Finalmente fueron identificadas algunas acciones humanas que amenazan la conservación de aves en el Seno de Reloncaví.

Palabras clave: Ciclo mareal, hora del día, variabilidad estacional, conservación

\section{INTRODUCCIÓN}

La Convención Relativa a los Humedales de Importancia Internacional (RAMSAR) define a los humedales como "extensiones de marismas, pantanos y turberas, o superficies cubiertas de aguas, sean éstas de régimen natural o artificial, permanentes o temporales, estancadas o corrientes, dulces, salobres o saladas, incluidas las extensiones de agua marina cuya profundidad en marea baja no exceda de seis metros" (Muñoz-Pedreros \& Möller 1997). Los humedales se cuentan entre los ecosistemas más productivos del planeta y han brindado sustento alimenticio, estético y cultural a varias civilizaciones del mundo (Montoya 2000). Estos sistemas ofrecen un buen refugio y abundante alimento a las aves acuáticas, además muchos de estos ambientes son considerados como importantes áreas de concentración para las aves migratorias (Blanco 1999).

El borde costero del sur de Chile se caracteriza por ser una extensa zona geográfica compuesta por estuarios, canales, islas y mares interiores (Silva et al. 1997), así como también por ser una zona rica en humedales (Collantes \& Faggi 1999). Desafortunadamente, uno de los principales problemas que enfrenta la conservación de los humedales en Chile es la carencia de información básica 
y aplicada, fundamental para orientar el manejo sustentable de estos ecosistemas (CONAMA 2005), ante ello, los monitoreos sobre las comunidades de aves presentes en los humedales pueden ayudar a contrarrestar dicha carencia de conocimientos, debido a que la avifauna es considerada un buen indicador de la condición ambiental de un ecosistema (Milesi et al. 2002).

Debido al escaso número de estudios realizados sobre aves acuáticas en humedales marinos del sur de Chile (40$55^{\circ} \mathrm{S}$ ), en especial considerando la abundancia de humedales en estas regiones (Victoriano et al. 2006), es necesario aumentar el esfuerzo de monitoreo en estos ambientes, que incluyan resultados de los posibles factores físicos que pueden afectar la actividad de las aves y por ende su presencia en estos humedales, tal como la hora del día, estación climática (Bibby et al. 1998) y el ciclo de mareas (Blanco 1998). Por lo anterior, el objetivo de este trabajo fue determinar el efecto del ciclo de mareas, la hora del día y la estación climática sobre la diversidad del ensamble de aves presentes en un humedal marino del sur de Chile.

\section{MATERIAL Y MÉtodos}

\section{ÁREA DE ESTUdIO}

El estudio se llevó a cabo en el humedal marino de Bahía Lenca (41 ${ }^{\circ} 36^{\prime} \mathrm{S}, 72^{\circ} 41^{\prime} \mathrm{W}$ ), localizado $33 \mathrm{~km}$ al este de la ciudad de Puerto Montt, en el Seno de Reloncaví, sur de Chile (Fig. 1). El Seno de Reloncaví es considerado la entrada portuaria hacia la zona patagónica austral del sur de Chile (Soto-Mardones et al. 2009). En este mar interior existen abundantes sistemas estuarinos donde predominan las formaciones vegetales halófilas conocidas como marismas, estas formaciones se desarrollan en condiciones extremas de alta salinidad y de anegamiento por efecto de las amplias fluctuaciones de marea existentes en la zona, debido a los hundimientos de terreno provocados por los sismos de mayo de 1960 (San Martín \& Ramírez 2002).

La costa de Bahía Lenca posee una playa de 1,3 km de longitud, constituida por diferentes sustratos como arena y piedras, con una marisma de aproximadamente 1,8 há y la desembocadura del río Lenca en el sector oriente de la playa. Durante la bajamar queda despejado un extenso

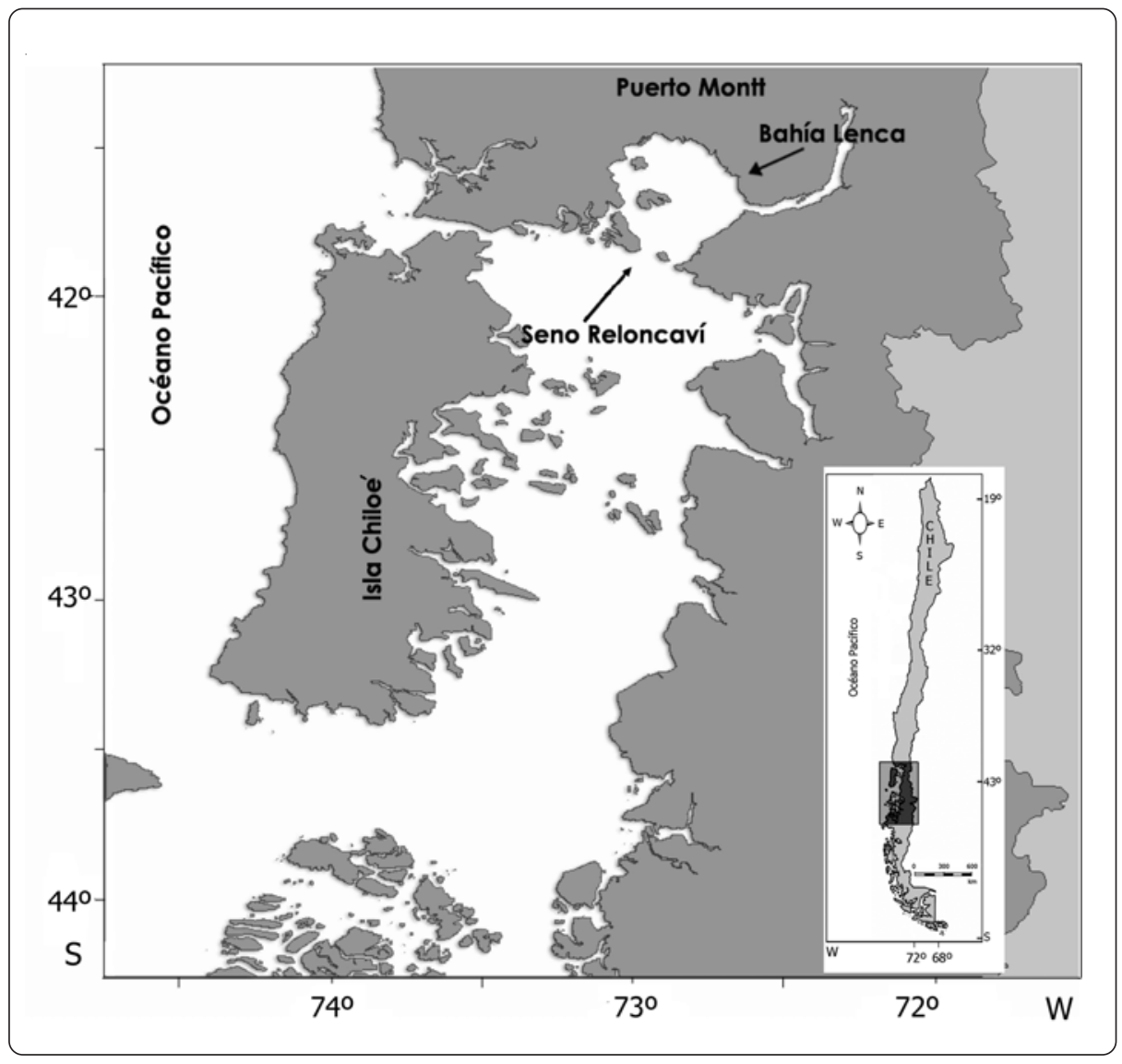

Figura 1. Ubicación geográfica del humedal marino de Bahía Lenca en el Seno de Reloncaví, sur de Chile / Geographic location of marine wetland of Lenca Bay, Reloncaví Sound, southern Chile 
suelo intermareal que alterna en forma muy heterogénea con sustratos blandos y duros, con una superficie aproximada de 26 há y una fauna representada por mitílidos, navajuelas y pequeños invertebrados. También existen áreas de repoblamiento del alga Gracillaria verrucosa (Hudson). La amplitud promedio de marea registrada en esta zona es de 7,46 m, donde la mayor parte de la variabilidad del nivel del mar está asociada a la marea (Soto-Mardones et al. 2009).

\section{OBTENCIÓN DE LOS DATOS}

Durante la primavera de 2006 y hasta el invierno de 2007 se estudió la composición del ensamble de aves que utilizaron el ambiente intermareal y de aguas someras en Bahía Lenca. El sitio de muestreo fue visitado mensualmente durante septiembre y noviembre del año 2006, enero, febrero, mayo, junio, julio y agosto del año 2007. En cada visita un observador realizó un conteo directo (sensu Bibby et al. 1998) del número de individuos de todas las especies de aves presentes en un área rectangular (30 há) que abarcó 1 km sobre la línea de costa y que se extendió $300 \mathrm{~m}$ desde la zona supramareal hacia mar adentro, desplazándose dentro de ella mediante caminata sigilosa en función de las obstrucciones geográficas propias de la planicie litoral que impedían cubrir visualmente la totalidad del área de estudio. El tiempo promedio de cada observación fue de $25 \pm 3 \mathrm{~min}$, totalizando 672 min de observación. Se registró también a las aves en vuelo no mayor a $5 \mathrm{~m}$ de altura.

Se establecieron tres horarios diurnos de observación en función de la variación en la duración del día y la latitud del sitio de estudio. Estos horarios fueron definidos como mañana (entre 07:00 y 09:00 h), medio día (entre 13:00 y 15:00 h) y tarde (entre 17:00 y 19:00 h). Los conteos fueron realizados en cada uno de estos horarios de observación, tanto en momentos de marea alta como de marea baja, definidos como los máximos y mínimos valores de altura del nivel del mar, respectivamente (SHOA 2006, 2007) ${ }^{1}$. Para cada estación climática se realizó una observación extensa que abarcó un ciclo mareal completo, registrando la secuencia de reemplazo de especies presentes en el sitio de estudio. En los momentos de marea alta aproximadamente el 90\% de la superficie del área de estudio quedó cubierta por el agua de mar. Las observaciones se realizaron utilizando un binocular $(8 \mathrm{x}$
30) y telescopio (45x), sin realizar conteos durante días con lluvia.

\section{ANálisis de Los Datos}

Los registros mensuales de riqueza y abundancia de aves fueron agrupados según la estación anual en la que fueron realizados primavera 2006 (septiembre y noviembre), verano 2007 (enero y febrero), otoño 2007 (mayo y junio) e invierno 2007 (julio y agosto), en donde la abundancia estacional por especie correspondió al número máximo de individuos registrados en cada conteo, para cada estación anual (Palacios et al. 1991). También se calculó la riqueza específica estacional como el número total de especies registradas en los conteos agrupados por estación climática. Para realizar comparaciones estacionales de los valores de riqueza específica se estandarizó el tamaño de la muestra mediante el método de rarefacción (Moreno 2001), utilizando el programa Biodiversity Professional Beta 1 (McAleece et al. 1997). La diversidad de especies se calculó utilizando el índice de Shannon-Wiener (H') (Moreno 2001), empleando logaritmos de base 10.

Se determinó la frecuencia de ocurrencia relativa (FR) de cada especie, la cual correspondió al número de conteos en que cada especie estuvo presente respecto al total de conteos realizados, expresando su valor en porcentaje (Gatto et al. 2005). Se utilizó un Índice de Importancia Relativa (IIR), modificado a partir del índice utilizado por Gatto et al. (2005) en humedales marinos de Argentina, como estimador general de la importancia de cada especie en el área de estudio, según la expresión IIR= 100(Ni/ $\mathrm{Nt}$ (Mi/Mt), donde $\mathrm{Ni}$ es la suma de las abundancias estacionales de la especie $i$ durante todo el período de estudio, Nt es el valor de abundancia total de aves durante todo el período de estudio, Mi es el número de conteos en los que la especie $i$ estuvo presente y Mt es el número total de conteos. Echevarría \& Chani (2000) recomiendan considerar como especie relevante para el ensamble de aves acuáticas a toda aquella que presente valores del IIR superiores a 0,5 .

La influencia del ciclo de marea sobre la diversidad de aves, fue evaluada mediante la versión de la prueba de $\mathrm{t}$ propuesta por Hutcheson (Moreno 2001), con la cual se determinaron diferencias pareadas entre los valores de diversidad obtenidos en los conteos realizados en momentos de marea alta y de marea baja. La prueba de

${ }^{1}$ SHOA. 2006, 2007. Tablas de marea de la costa de Chile. Servicio Hidrográfico y Oceanográfico de la Armada de Chile, Valparaíso. [en línea] $<w w w . s h o a . c l>~$ 
Hutcheson es análoga a la prueba t de Student y su propósito es comparar dos índices de diversidad y sus varianzas tanto espacial o temporalmente (Zar 1984). Los valores de diversidad para cada horario de observación, así como también los valores de abundancia estacional, fueron evaluados mediante pruebas de Kruskall-Wallis, con un nivel de significación $(\alpha)$ de 0,05, utilizando el programa estadístico SYSTAT 8.0 (Wilkinson 1998), ya que los valores no se distribuyeron normalmente (ShapiroWilk; $P<0,05)$. Para la clasificación taxonómica y el nombre común de cada especie de ave se utilizó la Lista Patrón de las Aves Chilenas (Araya et al. 1995).

\section{Resultados}

\section{CARACTERIZACIÓN DEL ENSAMBLE}

Durante el período de estudio se observó un total de 2502 aves pertenecientes a 35 especies y 19 familias (Tabla 1). La familia Laridae presentó la mayor riqueza de especies (4), seguida por las familias Anatidae y Scolopacidae que estuvieron representadas por tres especies (Tabla 1). La gaviota dominicana estuvo presente en el $100 \%$ de los censos, seguida por el pilpilén, el cisne de cuello negro, el zarapito, el pato quetru no volador, el queltehue y la gaviota cahuil, que presentaron valores de frecuencia de ocurrencia relativa (FR) mayores al 80\% (Tabla 1 ). Los valores más bajos, menores al 5\% de FR, correspondieron a la huala, el piquero, el cormorán imperial, la bandurria, la dormilona tontita, el chincol y el jilguero (Tabla 1).

La gaviota dominicana, la gaviota cahuil y el cisne de cuello negro presentaron los mayores valores de importancia relativa (IIR), seguidas por el zarapito, la gaviota de Franklin, el yeco, el pato quetru no volador, la golondrina chilena, el pilpilén, el tiuque, el chorlo chileno, el queltehue, el jote de cabeza negra y el playero de Baird, considerándose a estas especies como relevantes para el ensamble de aves debido a que presentaron valores del IIR superiores a 0,5 (Tabla 1 ).

\section{Cambios estacionales en el ENSAMble de aVes}

El mayor valor de riqueza específica estacional fue registrado durante el verano, mientras que en otoño se obtuvieron el menor valor de riqueza de especies (Tabla 2, Fig. 2). Pese a que no se registraron diferencias significativas entre los valores de abundancia estacional (Kruskal-Wallis $\mathrm{H}_{3}=0,392, P>0,50$, Tabla 2, Fig. 2), el mayor valor fue registrado en verano, en tanto que el menor número de individuos fue observado en invierno. Del total de especies, 14 de ellas fueron observadas durante las

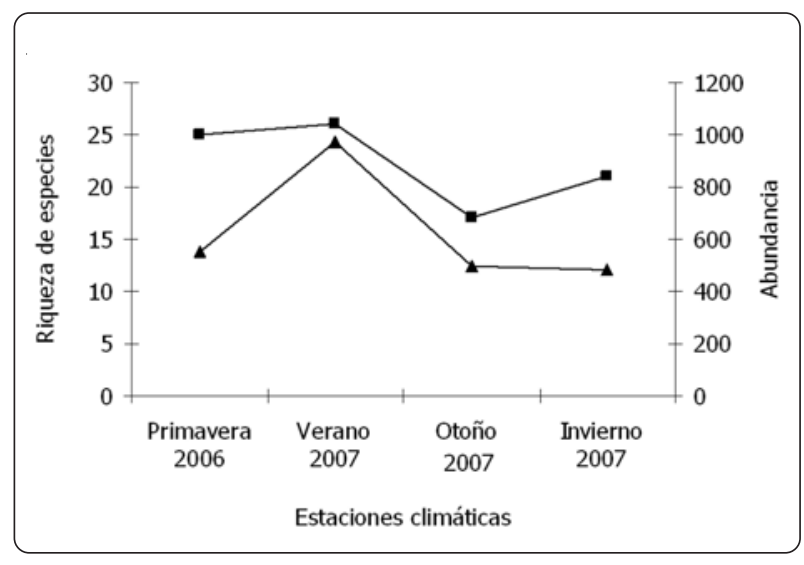

Figura 2. Variación estacional de la riqueza de especies (cuadrados) y la abundancia de aves (triángulos) en el humedal marino de Bahía Lenca, Seno de Reloncaví / Seasonal variation of species richness (squares) and abundance of birds (triangles) in the marine wetland of Lenca Bay, Reloncaví Sound

cuatro estaciones climáticas, mientras que siete especies fueron registradas solo durante la temporada estival, destacando la alta abundancia de la gaviota de Franklin y la golondrina chilena, en tanto que el chorlo chileno, la garza chica y la dormilona tontita tuvieron presencia exclusiva en la temporada invernal (Tabla 1).

\section{EFECTOS DEL CICLO MAREAL Y HORARIO DEL DÍA}

Los índices de diversidad fueron significativamente mayores durante marea baja, con mayor notoriedad durante la estación climática de otoño $\left(\mathrm{t}_{214}=6,704, P<0,0001\right.$, Tabla 2). No hubo diferencias significativas en la diversidad de aves entre los tres horarios de observación, durante todo el período de estudio (Kruskal-Wallis $\mathrm{H}_{2}=$ 0,368, $P>0,50$, Tabla 2).

\section{SECUENCIA DE REEMPLAZO DE ESPECIES EN FUNCIÓN DEL} CICLO MAREAL

En los momentos de marea alta se observó la presencia de aves buceadoras (yeco) y nadadoras (cisne de cuello negro) en la zona intermareal, muy cercanas a la orilla. En las rocas se posó el martín pescador, ave proveniente del sistema ribereño que desemboca en esta planicie, mientras que las aves pertenecientes al Orden Charadriformes se observaron segregadas en la zona supramareal. En función de la disminución de la altura de marea, las aves Charadriformes se desplazaron a la zona intermareal, mientras que las aves nadadoras y buceadoras se alejaron de la zona siguiendo la recogida del mar. Durante los momentos de marea baja se observó que las aves 
Tabla 1. Frecuencia de ocurrencia relativa (FR\%), valores del índice de importancia relativa (IIR) y la presencia temporal de las especies de aves registradas en distintas estaciones climáticas en el humedal marino de Bahía Lenca, Seno de Reloncaví; pr: primavera, ve: verano, ot: otoño, in: invierno / Relative frequency of occurrence (FR\%), values of the index of relative importance (IIR) and the temporal presence of each bird species recorded during different seasons in the marine wetland of Lenca Bay, Reloncaví Sound; pr: spring, ve: summer, ot: autumn, in: winter

\begin{tabular}{|c|c|c|c|c|}
\hline Especie & Nombre común & FR (\%) & IIR & Presencia \\
\hline \multicolumn{5}{|l|}{ Podicipedidae } \\
\hline Podiceps major (Boddaert, 1783) & Huala & 4,16 & 0 & ve \\
\hline \multicolumn{5}{|l|}{ Sulidae } \\
\hline Sula variegata (Tschudi, 1843) & Piquero & 4,16 & 0 & ot \\
\hline \multicolumn{5}{|l|}{ Pelecanidae } \\
\hline Pelecamus thagus (Molina, 1782) & Pelícano & 29,16 & 0,16 & $\mathrm{pr}$, in \\
\hline \multicolumn{5}{|l|}{ Phalacrocoracidae } \\
\hline Phalacrocorax brasilianus (Gmelin, 1789) & Yeco & 83,33 & 4,02 & $\mathrm{pr}$, ve, ot, in \\
\hline Phalacrocorax atriceps (King, 1828) & Cormorán imperial & 4,16 & 0 & in \\
\hline \multicolumn{5}{|l|}{ Ardeidae } \\
\hline Egretta thula (Molina, 1782) & Garza chica & 25,00 & 0,02 & ot, in \\
\hline Nycticorax nycticorax (Linné, 1758) & Huairavo & 12,50 & 0 & ve \\
\hline \multicolumn{5}{|l|}{ Threskiornithidae } \\
\hline Theristicus melanopis (Gmelin, 1789) & Bandurria & 4,16 & 0 & in \\
\hline \multicolumn{5}{|l|}{ Cathartidae } \\
\hline Coragyps atratus (Bechstein, 1783) & Jote de cabeza negra & 41,66 & 0,81 & $\mathrm{pr}$, ve, ot, in \\
\hline Cathartes aura (Linné, 1758) & Jote de cabeza colorada & 8,33 & 0,02 & $\mathrm{pr}$, ve \\
\hline \multicolumn{5}{|l|}{ Anatidae } \\
\hline Cygnus melanocorypha (Molina, 1782) & Cisne cuello negro & 87,50 & 11,04 & $\mathrm{pr}$, ve, ot, in \\
\hline Anas flavirostris (Vieillot, 1816) & Pato jergón chico & 12,50 & 0,18 & $\mathrm{pr}, \mathrm{ve}$, ot \\
\hline Tachyeres pteneres (Forster, 1844) & Quetru no volador & 87,50 & 1,98 & $\mathrm{pr}$, ve, ot, in \\
\hline \multicolumn{5}{|l|}{ Falconidae } \\
\hline Polyborus plancus (Miller, 1777) & Traro & 29,16 & 0,06 & $\mathrm{pr}$, ve, ot, in \\
\hline Milvago chimango (Vieillot, 1816) & Tiuque & 66,70 & 1,32 & $\mathrm{pr}$, ve, ot, in \\
\hline \multicolumn{5}{|l|}{ Haematopodidae } \\
\hline Haematopus palliatus (Temminck, 1820) & Pilpilén & 91,66 & 1,67 & $\mathrm{pr}$, ve, ot, in \\
\hline Haematopus ater (Vieillot \& Oudart, 1825) & Pilpilén negro & 41,66 & 0,41 & $\mathrm{pr}$, ve, ot, in \\
\hline \multicolumn{5}{|l|}{ Charadriidae } \\
\hline Vanellus chilensis (Molina, 1782) & Queltehue & 83,33 & 0,82 & $\mathrm{pr}$, ve, ot, in \\
\hline Charadrius modestus (Lichtenstein, 1823) & Chorlo chileno & 41,66 & 0,86 & ot, in \\
\hline \multicolumn{5}{|l|}{ Scolopacidae } \\
\hline Numenius phaeopus (Linné, 1758) & Zarapito & 87,50 & 5,69 & $\mathrm{pr}$, ve, ot, in \\
\hline Calidris alba (Pallas, 1764) & Playero blanco & 16,66 & 0,35 & $\mathrm{pr}, \mathrm{ve}$ \\
\hline Calidris bairdii (Coues, 1861) & Playero de Baird & 33,33 & 0,7 & pr, ve \\
\hline \multicolumn{5}{|l|}{ Laridae } \\
\hline Larus maculipennis (Lichtenstein, 1823) & Gaviota cahuil & 83,33 & 10,51 & $\mathrm{pr}$, ve, ot, in \\
\hline Larus pipixcan (Wagler, 1831) & Gaviota de Franklin & 29,16 & 5,07 & $\mathrm{pr}, \mathrm{ve}$ \\
\hline Larus dominicamus (Lichtenstein, 1823) & Gaviota dominicana & 100 & 19,29 & $\mathrm{pr}$, ve, ot, in \\
\hline Sterna hirundinacea (Lesson, 1831) & Gaviotin sudamericano & 12,50 & 0,14 & ve \\
\hline \multicolumn{5}{|l|}{ Alcedinidae } \\
\hline Ceryle torquata (Linné, 1766) & Martin pescador & 16,66 & 0,01 & $\mathrm{pr}$, ot, in \\
\hline \multicolumn{5}{|l|}{ Furnariidae } \\
\hline Cinclodes patagonicus (Gmelin, 1789) & Churrete & 62,50 & 0,41 & $\mathrm{pr}$, ve, ot, in \\
\hline \multicolumn{5}{|l|}{ Tyrannidae } \\
\hline Muscisaxicola macloviana (Garnot, 1829) & Dormilona tontita & 4,16 & 0 & in \\
\hline Lessonia rufa (Gmelin, 1789) & Colegial & 29,16 & 0,07 & $\mathrm{pr}$, ve, in \\
\hline \multicolumn{5}{|l|}{ Hirundinidae } \\
\hline Tachycineta meyeni (Cabanis, 1850) & Golondrina chilena & 45,83 & 1,77 & $\mathrm{pr}$, ve \\
\hline Pygochelidon cyanoleuca (Vieillot, 1817) & Golondrina de dorso negro & 25 & 0,06 & $\mathrm{pr}$, ve \\
\hline \multicolumn{5}{|l|}{ Emberizidae } \\
\hline Sicalis luteiventris (Meyen, 1834) & Chirihue & 20,83 & 0,08 & ve \\
\hline Zonotrichia capensis (Müller, 1776) & Chincol & 4,16 & 0 & $\mathrm{pr}$ \\
\hline \multicolumn{5}{|l|}{ Fringillidae } \\
\hline Carduelis barbata (Molina, 1782) & Jilguero & 4,16 & 0 & $\mathrm{pr}$ \\
\hline
\end{tabular}


Tabla 2. Abundancia estacional (N), riqueza de especies observada (Ro), riqueza de especies esperada según rarefacción $(\mathrm{Re})$ y valores del índice de diversidad ( $\left.\mathrm{H}^{\prime}\right)$ para el ensamble de aves registrado en diferentes períodos de marea y distintos horarios de observación, durante las cuatro estaciones climáticas en el humedal marino de Bahía Lenca, Seno de Reloncaví / Seasonal abundance (N), observed species richness (Ro), expected species richness according to rarefaction $(\mathrm{Re})$ and values diversity index $\left(\mathrm{H}^{\prime}\right)$ for the assemblage of birds recorded in different tidal periods and different times of observation, during the four seasons in the marine wetland of Lenca Bay, Reloncaví Sound

\begin{tabular}{|c|c|c|c|c|c|c|}
\hline Estación anual & Horario & Marea & $\mathrm{N}$ & Ro & $\operatorname{Re}$ & $\mathrm{H}^{\prime}$ \\
\hline \multirow[t]{6}{*}{ Primavera 2006} & mañana & alta & 274 & 15 & 14,98 & 0,81 \\
\hline & & baja & 158 & 13 & 12,95 & 0,83 \\
\hline & medio día & alta & 194 & 8 & 7,95 & 0,41 \\
\hline & & baja & 244 & 17 & 16,99 & 0,75 \\
\hline & tarde & alta & 74 & 15 & 14,96 & 1,07 \\
\hline & & baja & 243 & 14 & 13,98 & 0,77 \\
\hline \multirow[t]{6}{*}{ Verano 2007} & mañana & alta & 500 & 16 & 15,93 & 0,64 \\
\hline & & baja & 785 & 21 & 20,97 & 0,63 \\
\hline & medio día & alta & 545 & 12 & 11,99 & 0,59 \\
\hline & & baja & 398 & 15 & 14,96 & 0,7 \\
\hline & tarde & alta & 263 & 15 & 14,98 & 0,86 \\
\hline & & baja & 729 & 19 & 18,98 & 0,77 \\
\hline \multirow[t]{6}{*}{ Otoño 2007} & mañana & alta & 199 & 10 & 9,88 & 0,51 \\
\hline & & baja & 239 & 12 & 11,97 & 0,83 \\
\hline & medio día & alta & 206 & 10 & 9,99 & 0,73 \\
\hline & & baja & 162 & 14 & 13,99 & 0,87 \\
\hline & tarde & alta & 62 & 6 & 5,98 & 0,51 \\
\hline & & baja & 357 & 10 & 9,97 & 0,66 \\
\hline \multirow[t]{6}{*}{ Invierno 2007} & mañana & alta & 192 & 15 & 14,99 & 0,93 \\
\hline & & baja & 283 & 13 & 12,99 & 0,84 \\
\hline & medio día & alta & 165 & 10 & 9,95 & 0,71 \\
\hline & & baja & 118 & 12 & 11,82 & 0,85 \\
\hline & tarde & alta & 281 & 12 & 11,96 & 0,74 \\
\hline & & baja & 286 & 14 & 13,95 & 0,81 \\
\hline
\end{tabular}

Charadriformes aumentaron su actividad alimenticia, registrándose el ingreso a la planicie intermareal de queltehues, tiuques, traros y otras aves consideradas como terrestres. Al comenzar a subir el nivel de la marea se retiraron las aves terrestres, las aves Charadriformes se segregaron en su distribución en la zona intermareal y nuevamente las aves buceadoras y nadadoras volvieron al sitio de estudio.

\section{Discusión}

\section{CARACTERIZACIÓN DEL ENSAMBLE}

La gaviota dominicana, la gaviota cahuil y el cisne de cuello negro, presentaron los mayores valores de importancia relativa (IIR) para el ensamble de aves presente en el humedal marino de Bahía Lenca. Las dos primeras especies también obtuvieron los valores más altos de IIR en la zona 
intermareal y de aguas someras de Bahía de los Vientos en Argentina (García \& Gómez-Laich 2007), coincidiendo nuevamente el alto valor de IIR de la gaviota dominicana en el humedal marino de caleta Malaspina en Argentina (Gatto et al. 2005). Dicha gaviota se distribuye ampliamente en el sur de Sudamérica y es considerada un importante depredador de la zona intermareal (Silva et al. 2000), su conducta oportunista y su adaptación a ambientes antropizados ha generado un aumento en la abundancia y distribución geográfica de sus poblaciones durante los últimos años (Yorio et al. 1998). La gaviota cahuil también se distribuye ampliamente en el sur de Sudamérica (Del Hoyo et al. 1996). En Chile, esta gaviota habita ambientes costeros, interiores y zonas urbanas (González-Acuña et al. 2004, Araya \& Millie 2005, Cursach \& Rau 2008), nidificando generalmente en cuerpos de aguas continentales (Guicking et al. 2001).

El cisne de cuello negro es la tercera especie relevante para el ensamble de aves del humedal marino de Bahía Lenca. Esta ave es considerada emblemática de los humedales en Chile (Figueroa-Fábrega et al. 2006), distribuyéndose desde la región de Atacama $\left(34^{\circ} \mathrm{S}\right)$ hasta la Tierra del Fuego $\left(54^{\circ} \mathrm{S}\right)$ (Araya \& Millie 2005). Este cisne es un consumidor primario oportunista que se alimenta de plantas acuáticas dominantes en ecosistemas dulceacuícolas y marinos (Schlatter 1991, FigueroaFábrega et al. 2006), así como también se ha observado alimentándose de los desechos provenientes de una planta faenadora de reses de abastos en el litoral de Puerto Natales (Rau 1980). Las poblaciones de este cisne en Chile presentan un patrón de abundancia relacionado al fenómeno ENSO, ya que durante la presencia de dicho fenómeno la abundancia de este cisne aumenta en humedales de la zona central del país, viéndose disminuida la abundancia de sus poblaciones en la zona sur, ocurriendo todo lo contrario durante los períodos de sequía (interENSO) (Schlatter et al. 2002, Vilina et al. 2002). Esto coincide con la alta abundancia registrada para dicha especie en el humedal marino de Bahía Lenca, cuyas observaciones fueron realizadas durante un período interENSO (años 2006 y 2007).

\section{Cambios estacionales en el ensamble de aVes}

En el área de estudio, los mayores valores de riqueza de especies y abundancia de individuos fueron registrados durante el período estival, similar a lo registrado en humedales interiores de Chile (González-Acuña et al. 2004). Pese a ello, en el humedal marino de la localidad de Quillaipe-Metri (distante a $12 \mathrm{~km}$ al oeste de Bahía
Lenca), se registró un total de 20 especies de aves acuáticas durante verano e invierno del año 1998 respectivamente, con una mayor abundancia de individuos en invierno (Espinosa 1999), mientras que durante el año 1999 en la misma localidad se registraron 15 especies en verano y 17 especies en invierno, con una mayor abundancia de individuos en verano (Espinosa 2000). Esta variación en el registro de riqueza y abundancia estacional de aves se debe a que la avifauna presente en el Seno de Reloncaví se compone en su mayoría por especies migratorias provenientes del hemisferio norte y de la región austral, que encuentran en esta zona el ambiente adecuado para descansar y alimentarse (Espinosa et al. 1987, von Meyer \& Espinosa 1998). De la riqueza total de especies observadas en el humedal marino de Bahía Lenca, el 28,6\% fueron aves migratorias de las cuales siete especies fueron registradas solo durante la temporada estival, en tanto que tres especies tuvieron presencia exclusiva en la temporada invernal. La gaviota de Franklin es un migrante boreal (sensu Araya \& Millie 2005) que presentó la mayor abundancia de individuos durante la estación de verano en Bahía Lenca, esta gaviota es común y abundante durante la temporada estival entre los 41 y $44^{\circ} \mathrm{S}$, registrando con el tiempo un aumento en la distribución latitudinal de esta especie hacia el sur de Chile (Marín \& Couve 2001). Por otra parte, el chorlo chileno es un migrante austral (sensu Araya \& Millie 2005) nidificante en el extremo sur de Chile (52-55º) (Kusch \& Marín 2004), que visitó el sitio de estudio durante la temporada otoño-invierno, lo que concuerda con el patrón general de distribución latitudinal de esta especie en Chile (Kusch \& Marín 2004), se le observó alimentarse intensamente en la zona supramareal e intermareal durante los períodos de marea baja.

\section{EFECTOS DEL CICLO MAREAL SOBRE LA DIVERSIDAD DEL ENSAMBLE}

El ciclo mareal ejerce una fuerte influencia en la diversidad de aves costeras, ya que el índice H' fue mayor durante marea baja. Generalmente las aves aprovechan la marea baja para alimentarse de las presas existentes en la zona intermareal, mientras que durante los momentos de marea alta la eficiencia de forrajeo de las aves disminuye considerablemente debido a que la zona intermareal queda físicamente inaccesible para las aves, obligándolas a concentrarse en sitios altos que utilizan como áreas de dormidero o a desplazarse hacia sitios alternativos de forrajeo (Blanco 1998, 1999, Alvarado \& Moreno 2001).

En Bahía Lenca, durante los momentos de marea baja se sumaron, junto a las aves típicamente costeras, las 
especies de los ensambles pertenecientes a los 'agroecosistemas' y sistemas de vegetación arbustiva colindantes al humedal marino, como el tiuque, el queltehue, la bandurria, el traro y aves Passeriformes, generando un aumento en la diversidad de aves observada en el sitio de estudio.

\section{EFECTOS DEL HORARIO DEL DÍA SOBRE LA DIVERSIDAD DEL} ENSAMBLE

Variaciones en los niveles de actividad y cambios en la conducta de las aves durante el transcurso del día, pueden causar diferencias en la detectabilidad de estas especies y por ende introducir sesgo en los resultados de los censos (Palmeirim \& Rabaça 1994). Sin embargo, en este estudio la diversidad de aves observadas en el humedal marino de Bahía Lenca, fue similar durante la mañana, mediodía y tarde. Resultados similares fueron encontrados en un ensamble de aves playeras estudiadas en lagunas costeras de Perú (Torres et al. 2006), a diferencia de los registros obtenidos por Alvarado \& Moreno (2001) en aves playeras presentes en un manglar de Costa Rica, observando que la abundancia total de individuos tuvo valores máximos en horas tempranas de la mañana (07:00-08:00 h) y una marcada disminución en horas de la tarde (14:00-17:00 h). Esta disminución en la abundancia de aves playeras en horas de la tarde se debe a que en dicho horario estas aves se alejan de los sitios de alimentación para dirigirse a los sitios de descanso (Irons 1998).

\section{Conservación de las aves en el Seno de Reloncaví}

Durante las últimas décadas, el borde costero del Seno de Reloncaví ha sufrido una destrucción gradual de sus ambientes (von Meyer 1996) debido principalmente a: (1) la construcción de habitaciones humanas hasta la misma línea de marea alta (von Meyer \& Espinosa 1998), (2) al sobrepastoreo de las marismas por ganado equino y bovino (Espinosa et al. 1987), (3) importantes alteraciones de la zona intermareal debido a la extracción de grandes cantidades de rocas y piedras utilizadas como material de nivelación para el proyecto de asfaltamiento de la Carretera Austral, (4) disminución de la vegetación arbórea costera que conforma el hábitat apropiado para la nidificación del pato quetru no volador en Bahía Lenca (Cursach \& Rau 2009) y (5) las diversas actividades de acuicultura realizadas en la zona. Dichas alteraciones en el ambiente han afectado de manera importante a la avifauna local, como lo fue el relevante cese de los eventos reproductivos del cisne de cuello negro (von Meyer 1996) y la considerable disminución en la abundancia del flamenco chileno, el zarapito boreal Limosa haemastica (Linné, 1758) y el chorlo dorado Pluviales dominicana (Müller, 1776) en el sector de Coihuín-Chamiza (distante a 23 km al oeste de Bahía Lenca), localidad considerada como una vasta zona de alimentación para las aves.

Por otro lado, se ha evidenciado el efecto antrópico en la diversidad de aves silvestres, debido a que existen viviendas cerca de las áreas del Seno de Reloncaví, cuya presencia las afectan de dos formas: primero, con la crianza de aves generalistas y omnívoras (Cursach \& Rau 2008) que influyen sobre la diversidad del ensamble de aves, disminuyendo la riqueza de especies salvajes; y segundo, la presencia de animales domésticos, que amenazan de alguna forma, la presencia de las aves en su ambiente natural (Espinosa et al. 1987, von Meyer \& Espinosa 1998). Todo lo anterior indica que la alteración del ambiente costero por causa antrópica ha generado deterioro del paisaje natural y pérdida de vida silvestre en el Seno de Reloncaví, siendo necesario implementar un programa comunal y gubernamental que regule las actividades humanas y garantice la protección del ambiente costero de esta zona, mediante un plan de ordenamiento del uso del borde costero y un asesoramiento técnico a las comunidades rurales garantizando el correcto desarrollo de éstas en armonía con su medio ambiente.

\section{Agradecimientos}

Al Programa de Magíster en Ciencias de la Universidad de Los Lagos. Al profesor Ms. Bernardo Iturra, Director de la Escuela Rural de Bahía Lenca (años 2006-2007). A los señores Ignacio Escalante y Dr. Yerko Vilina por la facilitación de Literatura. A dos revisores anónimos de la RBM\&O por sus comentarios y contribuciones a este escrito.

\section{LITERATURA CITADA}

Alvarado G \& L Moreno. 2001. Abundancia de especies y uso de salinas por aves acuáticas. Brenesia 55-56: 123134.

Araya B \& G Millie. 2005. Guía de campo de las aves de Chile, 406 pp. Editorial Universitaria, Santiago.

Araya B, M Bernal, R Schlatter \& M Sallaberry. 1995. Lista patrón de las aves chilenas, 35 pp. Editorial Universitaria, Santiago.

Bibby C, M Jones \& S Marsden. 1998. Bird surveys, expedition field techniques, 143 pp. Expedition Advisory Centre, Royal Geographical Society, London. 
Blanco D. 1998. Uso de hábitat por tres especies de aves playeras (Pluviales dominicana, Limosa haemastica y Calidris fuscicollis) en relación con la marea en Punta Rasa, Argentina. Revista Chilena de Historia Natural 71: 87-94.

Blanco D. 1999. Los humedales como hábitat de aves acuáticas. Boletín UNESCO, Uruguay: 208-217.

Collantes M \& A Faggi. 1999. Los humedales del sur de Sudamérica. Boletín UNESCO, Uruguay: 14-24.

CONAMA. 2005. Estrategia nacional de conservación y uso racional de los humedales en Chile, 30 pp. Gobierno de Chile, Santiago.

Cursach J \& J Rau. 2008. Influencia de las perturbaciones humanas sobre la diversidad del ensamble de aves costeras en el Seno de Reloncaví, sur de Chile. Boletín Chileno de Ornitología 14(2): 92-97.

Cursach J \& J Rau. 2009. Abundancia y nidificación del pato quetru no volador Tachyeres pteneres en Bahía Lenca, Seno de Reloncaví, sur de Chile. Boletín del Museo Nacional de Historia Natural, Chile 58: 91-94.

Del Hoyo J, A Elliot \& J Sargatal. 1996. Handbook of the birds of the world. Vol. 3. Hoatzin to Auks: 1-821. Lynx Edicions, Barcelona.

Echevarría A \& J Chani. 2000. Estructura de la comunidad de aves acuáticas del embalse El Cadillal, Tucumán, Argentina. Acta Zoológica Lilloana 45: 219-232.

Espinosa L. 1999. Censo Neotropical de aves acuáticas 1998. Boletín Chileno de Ornitología 6: 47-52.

Espinosa L. 2000. Censo de aves acuáticas 1999, Chile. Boletín Chileno de Ornitología 7: 39-47.

Espinosa L, M Sallaberry \& A von Meyer. 1987. Aves observadas en la zona de Chamiza y mar adyacente. Boletín Informativo Unión de Ornitólogos de Chile 4: 14-28.

Figueroa-Fabrega L, J Galaz \& C Merino. 2006. Conocimiento y conservación del cisne de cuello negro Cygnus melancoryphus (Molina, 1782) en el humedal del río Cruces, Valdivia, Chile. Gestión Ambiental 12: 77-89.

García G \& A Gómez-Laich. 2007. Abundancia y riqueza específica en un ensamble de aves marinas y costeras del sudeste de la provincia de Buenos Aires, Argentina. Hornero 22(1): 9-16.

Gatto A, F Quintana, PYorio \& N Lisnizer. 2005. Abundancia y diversidad de aves acuáticas en un humedal marino del golfo San Jorge, Argentina. Hornero 20(2): 141-152.

González-Acuña D, C Benavente \& R Figueroa. 2004. Avifauna de la laguna Santa Elena, región del Bío-Bío. Boletín Chileno de Ornitología 10: 13-18.

Guicking D, S Mickstein, P Becker \& R Schlatter. 2001. Nest site selection by brown-hooded gull (Larus maculipennis), trudeau's tern (Sterna trudeaui) and whitefaced ibis (Plegadis chihi) in a south Chilean tule marsh. Ornitología Neotropical 12: 285-296.
Irons D. 1998. Foraging area fidelity of individual seabirds in relation to tidal cycles and flock feeding. Ecology 79(2): 647-655.

Kusch A \& M Marín. 2004. Distribución del chorlo chileno, Charadrius modestus, (Lichtenstein) (Charadriidae) en Chile. Anales del Instituto de la Patagonia 32: 69-78.

Marín M \& E Couve. 2001. La gaviota de Franklin, Larus pipixcan (Laridae), al sur de Latitud $41^{\circ} \mathrm{S}$, con nuevos registros de distribución. Anales del Instituto de la Patagonia 29: 161-163.

McAleece N, PJD Lambshead \& GLJ Paterson. 1997. Biodiversity Pro. The Natural History Museum, London. [en línea] < http://www.sams.ac.uk/ >

Milesi F, L Marone, J Lopez de Casenave, V Cueto \& E Mezquida. 2002. Gremios de manejo como indicadores de las condiciones del ambiente: un estudio de caso con aves y perturbaciones del hábitat en el Monte central, Argentina. Ecología Austral 12: 149-161.

Montoya C. 2000. Aspectos legales vinculados a la conservación de los humedales en Chile. Gayana 64(1): 1-2.

Moreno C. 2001. Métodos para medir la biodiversidad. Vol. 1: 1-84. M\&T-Manuales y Tesis SEA, Zaragoza.

Muñoz-Pedreros A \& P Möller. 1997. Conservación de humedales. Bases para la conservación de humedales de Chile, 95 pp. Ediciones del Centro de Estudios Agrarios \& Ambientales (CEA), Valdivia.

Palacios E, A Escofet \& D Loya-Salinas. 1991. El estero de Punta Banda, B.C. México, como eslabón del "corredor del Pacífico": abundancia de aves playeras. Ciencias Marinas 17(3): 109-131.

Palmeirim J \& J Rabaça. 1994. A method to analyze and compensate for time-of-day effects on bird counts. Journal of Field Ornithology 65(1): 17-26.

Rau J. 1980. Fluctuación estacional de Cynus melancoryphus (Molina) en Puerto Natales (Última Esperanza, XII Región de Magallanes). Noticiario Mensual, Museo Nacional de Historia Natural, Chile 24: 279-280.

San Martín C \& C Ramírez. 2002. Sinecología de una marisma en el Seno de Reloncaví (Llanquihue, X Región, Chile). Revista Geográfica de Valparaíso 32-33: 307-319.

Schlatter R, A Salazar, A Villa \& J Meza. 1991. Reproductive biology of Black-necked Swan Cygnus melancoryphus at three Chilean wetland areas and feeding ecology at Rio Cruces. Wildfowl 1: 268-271.

Schlatter R, R Navarro \& P Corti. 2002. Effects of El Niño Southern Oscillations on numbers of Black-necked Swans at Río Cruces Sanctuary, Chile. Waterbirds 25: 114-122.

Silva M, R Bastida \& C Darrieu. 2000. Dieta de la gaviota cocinera (Larus dominicanus) en zonas costeras de la provincia de Buenos Aires, Argentina. Ornitología Neotropical 11: 331-339. 
Silva N, C Calvete \& H Sievers. 1997. Características oceanográficas físicas y químicas de canales australes chilenos entre Puerto Montt y laguna San Rafael (Crucero Cimar-Fiordo 1). Ciencia y Tecnología del Mar 20: 23106.

Silva P, CAP Andrade, VMFA Timoteo, E Rocha \& LMP Valente. 2006. Dietary protein, growth, nutrient utilization and body composition of juvenile blackspot seabream, Pagellus bogaraveo (Brunnich). Aquaculture Research 37: 1007-1014.

Soto-Mardones L, J Letelier, S Salinas, E Pinillas \& J Beldar. 2009. Análisis de parámetros oceanográficos y atmosféricos del Seno de Reloncaví. Gayana 73(1): 141155.

Torres M, Z Quinteros \& F Takano. 2006. Variación temporal de la abundancia y diversidad de aves limícolas en el refugio de vida silvestre Pantanos de Villa, Lima-Perú. Ecología Aplicada 5(1): 119-125.

Victoriano P, A González \& R Schlatter. 2006. Estado de conocimiento de las aves de aguas continentales de Chile. Gayana 70(1): 140-162.
Vilina Y, H Cofré, C Silva-García, M García \& C Pérez. 2002. Effects of El Niño on abundance and breeding of Black-necked Swans on El Yali wetland in Chile. Waterbirds 25: 123-127.

Von Meyer A. 1996. Nidificación del pato jergón chico (Anas flavirostris) en una zona poblada de la X Región. Boletín Chileno de Ornitología 3: 38-39.

Von Meyer A \& L Espinosa. 1998. Situación del flamenco chileno (Phoenicopterus chilensis) en Chiloé y sur de la Provincia de Llanquihue. Boletín Chileno de Ornitología 5: 16-20.

Wilkinson L. 1998. Systat 8.0. Statistics. SPSS, Chicago. [CDROM].

Yorio P, M Bertellotti, P Gandini \& E Frere. 1998. Kelp gulls (Larus dominicanus) breeding on the Argentine coast: population status and a review of its relationship with coastal management and conservation. Marine Ornithology 26: 11-18.

Zar J. 1984. Biostatistical analysis, 718 pp. Prentice Hall, New York.

Recibido el 11 de marzo de 2010 y aceptado el 31 de agosto de 2010 